\title{
On a Simple System of Discrete Two-Scale Difference Equations
}

\author{
L. Berg and M. Krüppel
}

\begin{abstract}
A special system of two discrete two-scale difference equations with polynomial solutions is investigated. For the solutions, addition and subtraction theorems are established showing in particular the behaviour of the solutions for a great argument, as well as further relations and inequalities. Also, corresponding generating functions are constructed which imply explicit representations for the solutions.
\end{abstract}

Keywords: Discrete two-scale difference equations, polynomials, addition theorems, generating functions, Collatz graph, Fibonacci numbers

AMS subject classification: $39 \mathrm{~A} 10,39 \mathrm{~A} 12,26 \mathrm{C} 05,30 \mathrm{C} 10$

\section{Introduction}

In this paper we consider the special system

$$
\left.\begin{array}{rl}
Z_{2 k} & =p Z_{k} \\
Z_{2 k+1} & =q Z_{k}+r Z_{k+1}
\end{array}\right\} \quad(k \dot{\mathbb{N}})
$$

of two discrete two-scale difference equations under the initial condition

$$
Z_{1}=1
$$

The coefficients are assumed to be non-vanishing complex numbers, and the solution is obviously a polynomial $Z_{n}=Z_{n}(p, q, r)$ of the coefficients. In a forthcoming paper [3] the solution of system (1.1) shall be used for an explicit representation of solutions of continuous two-scale difference equations at dyadic points. Such equations appear in wavelet theory and subdivision schemes, cf. [4,7]. The special case $S_{n}=Z_{n}(q+1, q, 1)$ was already considered in [2] in connection with de Rham's singular function. After replacement $Z_{n+1}=x_{n}$, the second equation of system (1.1) with $q=\frac{1}{c}$ and $r=-\frac{1}{c}$ for $c>0$ appeared also in $[1,5]$, however in another context and without its first equation.

It is very simple to calculate the first polynomials $Z_{n}$ (cf. Table 1 ) as well as $Z_{2^{\prime}}=p^{\ell}$ for $\ell \in \mathbb{N}_{0}$, but our aim is to analyze the general structure of $Z_{n}$ which becomes visible in addition and subtraction theorems. We establish further relations and calculate infinite series. For $p=q=r=1$. some $Z_{n}$ are the Fibonacci numbers which here have an

Both authors: FB Math. der Univ., Universitätspl. 1, D-18051 Rostock 
extremal property. Moreover, we construct generating functions of $Z_{n}$ and of related polynomials and derive different explicit representations for $Z_{n}$.

\begin{tabular}{l|l}
$n$ & $Z_{n}$ \\
\hline 1 & 1 \\
\hline 2 & $p$ \\
\hline 3 & $p r+q$ \\
4 & $p^{2}$ \\
\hline 5 & $p r^{2}+(p+r) q$ \\
6 & $p^{2} r+p q$ \\
7 & $p r(p+q)+q^{2}$ \\
8 & $p^{3}$ \\
\hline
\end{tabular}

\begin{tabular}{r|l}
$n$ & $Z_{n}$ \\
\hline 9 & $p r^{3}+\left(p^{2}+p r+r^{2}\right) q$ \\
10 & $p^{2} r^{2}+p q(p+r)$ \\
11 & $p r^{2}(p+q)+(p q+p r+q r) q$ \\
12 & $p^{3} r+p^{2} q$ \\
13 & $p r(p q+p r+q r)+(p+r) q^{2}$ \\
14 & $p^{2} r(p+q)+p q^{2}$ \\
15 & $p r\left(p^{2}+p q+q^{2}\right)+q^{3}$ \\
16 & $p^{4}$ \\
\hline
\end{tabular}

Table 1: The first polynomials $Z_{n}=Z_{n}(p, q, r)$

In Table 1 it is conspicuous that the non-vanishing coefficients of the polynomials $Z_{n}$ are all equal to 1 . However, this is not a general property as the example

$$
Z_{21}=p r^{2}(p q+p r+q r)+\left(p^{2} q+2 p q r+p r^{2}+q r^{2}\right) q
$$

shows. It is possible to use the second equation of system (1.1) also for $k=0$ and to introduce

$$
Z_{0}=\frac{1-r}{q}
$$

However, only in the case $p=1$ or $r=1$, i.e.

$$
(p-1)(r-1)=0
$$

the first equation of system (1.1) is compatible with value (1.3) so that we shall use (1.3) only in these two cases.

\section{Addition theorems}

We begin with the construction of addition theorems, i.e. of formulas for $Z_{n}$ where $n$ is a certain sum of two terms.

Proposition 2.1. Under conditions (1.2) and $p \neq r$ the solution of system (1.1) with initial condition (1.2) has the structure

$$
Z_{2 \ell k+j}=\frac{q}{p-r}\left(p^{\ell} X_{j}-r^{\ell} Y_{j}\right) Z_{k}+r^{\ell} Y_{j} Z_{k+1}
$$

for $0 \leq j \leq 2^{\ell} \quad\left(k \in \mathbb{N} ; j, \ell \in \mathbb{N}_{0}\right)$ where $X_{j}=Z_{j}\left(1, \frac{q}{p}, \frac{r}{p}\right)$ and $Y_{j}=Z_{j}\left(\frac{p}{r}, \frac{q}{r}, 1\right)$.

Proof. In the case $\ell=0$ equation (2.1) is satisfied for $j \in\{0,1\}$ in view of $X_{0}=$ $\frac{p-r}{q}, Y_{0}=0$ and $X_{1}=Y_{i}=1$. Hence for $\ell=0$ and $0 \leq j \leq 1$ the polynomials $Z_{n}$ have the structure

$$
Z_{2^{\ell} k+j}=X_{\ell j} Z_{k}+Y_{\ell j} Z_{k+1}
$$


We assume that (2.2) is satisfied for a fixed $\ell$ and $0 \leq j \leq 2^{\ell}$. Replacing $k$ by $2 k$ und using (1.1) we obtain

$$
Z_{2^{\ell+1 k+j}}=\left(p X_{\ell j}+q Y_{\ell j}\right) Z_{k}+r Y_{\ell j} Z_{k+1}
$$

and therefore

$$
\left.\begin{array}{rl}
X_{\ell+1, j} & =p X_{\ell j}+q Y_{\ell j} \\
Y_{\ell+1, j} & =r Y_{\ell j}
\end{array}\right\} .
$$

Analogously, replacing $k$ in $(2.2)$ by $2 k+1$ we find

$$
\left.\begin{array}{rl}
X_{\ell+1,2^{\ell}+j} & =q X_{\ell j} \\
Y_{\ell+1,2^{\ell}+j} & =r X_{\ell j}+p Y_{\ell j}
\end{array}\right\}
$$

both equations for $0 \leq j \leq 2^{\ell}$. This shows that (2.2) is satisfied for $\ell+1$ instead of $\ell$ and for $0 \leq j \leq 2^{\ell+1}$. Hence by induction (2.2) is proved for all $\ell \in \mathbb{N}_{0}$.

Equations (2.3) have the general solutions

$$
\left.\begin{array}{rl}
X_{\ell j} & =\frac{q}{p-r}\left(p^{\ell} X_{j}-r^{\ell} Y_{j}\right) \\
Y_{\ell j} & =r^{\ell} Y_{j}
\end{array}\right\}
$$

for every fixed $j$ and $j \leq 2^{\ell}$ so that (2.2) implies (2.1). Replacing $j$ in (2.1) by $2 j$ and using $Z_{2^{\ell k+2 j}}=p Z_{2^{\ell-1} k+j}$ for $\ell \geq 1$ we obtain by comparison of coefficients

$$
\begin{aligned}
& X_{2 j}=X_{j} \\
& Y_{2 j}={ }_{r}^{e} Y_{j} .
\end{aligned}
$$

Analogously, replacing $j$ in (2.1) by $2 j+1$ and using $Z_{2^{\iota} k+2 j+1}=q Z_{2 \ell-1 k+j}+r Z_{2^{\ell-1} k+j+1}$ we obtain

$$
\begin{aligned}
X_{2 j+1} & ={ }_{p}^{q} X_{j}+r p X_{j+1} \\
Y_{2 j+1} & ={ }_{r}^{q} Y_{j}+Y_{j+1} .
\end{aligned}
$$

In view of the initial conditions the proposition is proved

\section{Remark 2.2.}

1. In the case (1.4) at most two of the sequences $X_{n}, Y_{n}, Z_{n}$ are different since $X_{n}=Z_{n}$ for $p=1$ and $Y_{n}=Z_{n}$ for $r=1$. Obviously, $X_{n}=Y_{n}=Z_{n}$ for $p=r=1$.

2. For $k=1$ equation (2.1) specializes to

$$
Z_{2^{l}+j}=\frac{q}{p-r} p^{l} X_{j}+\left(p-\frac{q}{p-r}\right) r^{l} Y
$$

with $0 \leq j \leq 2^{\ell}\left(\ell \in \mathbb{N}_{0}\right)$. In view of $Y_{j}=Z_{j}\left(\frac{p}{r}, \frac{q}{r}, 1\right)$ and $X_{j}=Z_{j}\left(1, \frac{q}{p}, \frac{r}{p}\right)$ equation (2.6) immediately implies

$$
\begin{aligned}
& Y_{2^{\ell}+j}=\frac{q}{p-r}\left(\frac{p}{r}\right)^{l} X_{j}+\left(\frac{p}{r}-\frac{q}{p-r}\right) Y_{j} \\
& X_{2^{\ell}+j}=\frac{q}{p-r} X_{j}+\left(1-\frac{q}{p-r}\right)\left(\frac{r}{p}\right)^{l} Y_{j}
\end{aligned}
$$


and the three equations (2.6) - (2.8) can be used to calculate $Z_{n}$ for $n=2^{\gamma_{k}}+\ldots+2^{\gamma_{1}}+2^{\gamma_{0}}$ with integers $\gamma_{k}>\ldots>\gamma_{1}>\gamma_{0} \geq 0$. In Section 6 we shall come back to this question in a special case. Equations (2.5), (2.7) - (2.8) can also be used to check equations (2.4).

3. Eliminating $X_{j}$ and $Y_{j}$ out of (2.6) - (2.8) we obtain the relation

$$
Z_{n}=\frac{1}{p-r}\left((p-1) r^{\ell+1} Y_{n}-(r-1) p^{\ell+1} X_{n}\right)
$$

for $2^{\ell} \leq n \leq 2^{\ell+1}\left(\ell \in \mathbb{N}_{0}\right)$.

The excluded case $p=r$ in Proposition 2.1 can be treated in an analogous way or by means of the limit process $r \rightarrow p$. For convenience, we consider the case $p \rightarrow r$ and write afterwards once inore $p$ instead of $r$. The appearing derivatives with respect to $p$ shall be labelled by means of a dash.

Proposition 2.3. For $p=r$ the solution of system (1.1) with initial condition (1.2) has the structure.

$$
Z_{2^{\ell}+j}=p^{\ell-1}\left[\left(\ell q+p^{2}\right) Y_{j}-q w_{j}\right]
$$

where

$$
w_{j}=\left.Z_{j}^{\prime}(p, x p, p)\right|_{p=1}
$$

satisfies

$$
w_{2^{\ell}+j}(x)=\left(x \ell^{2}+\ell+1\right) Y_{j}-\ell x w_{j}(x)
$$

for $0 \leq j \leq 2^{\ell}\left(j, \ell \in \mathbb{N}_{0}\right), Y_{j}=Z_{j}(1, x, 1), x=\frac{q}{p}$, and $w_{0}=-\frac{1}{x}, w_{1}=0$.

Proof. Since $Z_{n}$ is a polynomial in $p, q, r$ it is differentiable and so are $X_{n}$ and $Y_{n}$ in vicw of $p \neq 0$ and $r \neq 0$. Equation (2.6) can be written in the form

$$
Z_{2^{\ell}+j}=\left(p r^{\ell}+q \frac{p^{\ell}-r^{\ell}}{p-r}\right) Y_{j}-p^{\ell} q \frac{Y_{j}-X_{j}}{p-r}
$$

For $p \rightarrow r$ both $X_{j}=Z_{j}\left(1, \frac{q}{p}, \frac{r}{p}\right)$ and $\dot{Y}_{j}=Z_{j}\left(\frac{p}{r}, \frac{q}{r}, 1\right)$ converge to $Z_{j}\left(1, \frac{q}{p}, 1\right)$ and, by means of de l'Hospital's rule (which is also applicable to holomorphic functions), we obtain

$$
Z_{2^{\ell}+j}=p^{\ell-1}\left(p^{2}+\ell q\right) Y_{j}-p^{\ell} q\left(Y_{j}^{\prime}-X_{j}^{\prime}\right)
$$

and therefore (2:10) with

$$
w_{j}=\left.p\left[Z_{j}^{\prime}\left(\underset{r}{p}, \frac{q}{r}, 1\right)-Z_{j}^{\prime}\left(1, \frac{q}{p}, \frac{r}{p}\right)\right]\right|_{r=p}
$$

Obviously, $w_{j}$ depends on $x=\frac{q}{p}$ alone and it can be represented as (2.11). In particular, (2.11) yields the initial values of $w_{j}$ for $j=0$ and $j=1$. Substituting $q=p x$ in (2.10) we obtain

$$
Z_{2^{\ell}+j}=p^{\ell}\left[(\ell x+p) Y_{j}-x w_{j}\right]
$$

and by differentiation with respect to $p$, choosing $p=1$ and considering (2.11), we also have proved (2.12) 
Remark 2.4. More generally, it follows from (2.1) for $p \rightarrow r$

$$
Z_{2 k_{k+j}}=p^{\ell-1} q\left(\ell Y_{j}-w_{j}\right) Z_{k}+p^{\ell} Y_{j} Z_{k+1}
$$

and in view of (2.11)

$$
w_{2^{\ell} k+j}=x\left(\ell^{2} Y_{j}-\ell w_{j}\right) Y_{k}+\ell Y_{j} Y_{k+1}+x\left(\ell Y_{j}-w_{j}\right) w_{k}+Y_{j} w_{k+1}
$$

for $0 \leq j \leq 2^{\ell}\left(k \in \mathbb{N}, \ell \in \mathbb{N}_{0}\right), Y_{j}=Z_{j}(1, x, 1)$. For $j=0$ this implies $w_{2^{\ell} k}=\ell Y_{k}+w_{k}$ and for $\ell \geq 0$ in particular $w_{2 \ell}=\ell$. Moreover, for $\ell=1$ and $j=0$ resp. $j=1$ we easily see:

Corollary 2.5. The polynomials $w_{j}(j \in \mathbb{N})$ are uniquely determined by the initial value $w_{1}=0$ and the system

$$
\left.\begin{array}{rl}
w_{2 j} & =w_{j}+Y_{j} \\
w_{2 j+1} & =x w_{j}+w_{j+1}+Y_{2 j+1}
\end{array}\right\},
$$

$Y_{j}=Z_{j}(1, x, 1)$, which is the inhomogeneous counterpart to the homogeneous system (1.1) with $p=r=1$ and $q=x$.

By elimination of $Y_{j}$ in (2.16), using $Y_{2 j+1}=x Y_{j}+Y_{j+1}$, we obtain the further relation

$$
w_{2 j+1}=x w_{2 j}+w_{2 j+2}
$$

which is also satisfied by $Y_{n}$ instead of $w_{n}$, and from (2.10) and (2.12) with $p=1$ and $q=x$ we get

$$
w_{2^{\ell}+j}=\ell Y_{2^{\imath}+j}+Y_{j}
$$

$Y_{j}=Z_{j}(1, x, 1)$. All these relations can be checked for the first indices by means of Table 2.

\begin{tabular}{l|l|l}
$n$ & $Y_{n}$ & $w_{n}$ \\
\hline 1 & 1 & 0 \\
\hline 2 & 1 & 1 \\
\hline 3 & $1+x$ & $2+x$ \\
4 & 1 & 2 \\
\hline 5 & $1+2 x$ & $3+4 x$ \\
6 & $1+x$ & $3+2 x$ \\
7 & $1+x+x^{2}$ & $3+3 x+2 x^{2}$ \\
8 & 1 & 3 \\
\hline
\end{tabular}

\begin{tabular}{r|l|l}
$n$ & $Y_{n}$ & $w_{n}$ \\
\hline 9 & $1+3 x$ & $4+9 x$ \\
10 & $1+2 x$ & $4+6 x$ \\
11 & $1+2 x+2 x^{2}$ & $4+7 x+6 x^{2}$ \\
12 & $1+x$ & $4+3 x$ \\
13 & $1+2 x+2 x^{2}$ & $4+8 x+6 x^{2}$ \\
14 & $1+x+x^{2}$ & $4+4 x+3 x^{2}$ \\
15 & $1+x+x^{2}+x^{3}$ & $4+4 x+4 x^{2}+3 x^{3}$ \\
16 & 1 & 4 \\
\hline
\end{tabular}

Table 2: The first polynomials $Y_{n}=Z_{n}(1, x, 1)$ and $w_{n}(x)$ 


\section{Subtraction theorems}

There exist analogous formulas for negative $j$, i.e. corresponding subtraction theorems.

Proposition 3.1. In the case $p \neq q$ the solution of system (1.1) with initial condi. tion (1.2) has the property

$$
Z_{2^{\ell}-j}=\frac{r}{p-q} p^{\ell} U_{j}+\left(\frac{1-r}{q}-\frac{r}{p-q}\right) q^{\ell} V_{j}
$$

for $0 \leq j \leq 2^{\ell}-1\left(\ell \in \mathbb{N}_{0}\right)$ where $U_{j}=Z_{j}\left(1, \frac{r}{p}, \frac{q}{p}\right)$ and $V_{j}=Z_{j}\left(\frac{p}{q}, \frac{r}{q}, 1\right)$.

The proof can easily be carried out inductively using the initial values $Z_{2^{\ell}}=p^{\ell}$, $U_{0}=\frac{p-q}{r}, V_{0}=0$ and the recursions

$$
\left.\left.\begin{array}{rl}
U_{2 j} & =U_{j} \\
U_{2 j+1} & =\frac{r}{p} U_{j}+\frac{q}{p} U_{j+1}
\end{array}\right\} \quad \text { and } \quad \begin{array}{rl}
V_{2 j} & =\underset{q}{R} V_{j} \\
V_{2 j+1} & =\frac{r}{q} V_{j}+V_{j+1}
\end{array}\right\}
$$

so that it shall be omitted here. For $j=2^{\ell}$ the right-hand side of (3.1) is equal to $\frac{1-r}{q} p^{\ell}$, and is equal to (1.3) for all $\ell$ if and only if $p=1$ or $r=1$.

As a consequence of (2.6) and (3.1) we find

$$
Z_{2^{\ell}+j}=p r^{\ell} Z_{j}(\underset{r}{R}, \underset{r}{q}, 1)+q^{\ell} Z_{2^{\ell}-j}\left(\underset{q}{R}, \frac{r}{q}, 1\right)
$$

and this equation is not only valid for $0 \leq j<2^{\ell}$ but also for $j=2^{\ell}$. Owing to continuity, equation (3.2) remains valid in the limit case $p=r$. Since both terms on the right-hand side of (3.2) are homogeneous polynomials we can conclude (cf. Table 1):

Corollary 3.2. For $1 \leq j \leq 2^{\ell}-1$ every polynomial $Z_{2^{\ell}+j}$ is a sum of a homogeneous polynomial of degree $\ell+1$ plus such a polynomial of degree $\ell$.

It is also possible to consider the limit case $q \rightarrow p$ in (3.1) where we proceed analogously as before.

Proposition 3.3. For $p=q$ the solution of system (1.1) with initial condition (1.2) has the property

$$
Z_{2^{\ell}-j}=p^{\ell-1}\left[(r(\ell-1)+1) U_{j}-r w_{j}\right]
$$

for $0 \leq j \leq 2^{\ell}-1 \cdot\left(\ell \in \mathbb{N}_{0}\right)$ where $U_{j}=Z_{j}\left(1, \frac{r}{p}, 1\right)$ and $w_{j}=w_{j}\left(\frac{r}{p}\right)$ is determined by (2.16) with $x=\frac{r}{p}$ and $w_{1}=0$.

Proof. By means of de l'Hospital's rule we obtain from (3.1) for $p \rightarrow q$

$$
Z_{2^{\ell}-j}=(r \ell+1-r) p^{\ell-1} Z_{j}\left(1, \frac{r}{p}, 1\right)-\left.r p^{\ell}\left[Z_{j}^{\prime}\left(\frac{p}{q}, \frac{r}{q}, 1\right)-Z_{j}^{\prime}\left(1, \frac{r}{p}, \frac{q}{p}\right)\right]\right|_{q=p}
$$

where in view of $(2.11)$

$$
\left.p\left[Z_{j}^{\prime}\left(\frac{p}{q}, \frac{r}{q}, 1\right)-Z_{j}^{\prime}\left(1, \frac{r}{p}, \frac{q}{p}\right)\right]\right|_{q=p}=w_{j}\left(\frac{r}{p}\right)
$$

so that (3.3) is proved 
Analogously, in the case $x \neq 1$ we can derive

$$
w_{2^{\ell}-j}(x)=\frac{\ell}{1-x}\left(U_{j}-x^{\ell} V_{j}\right)-x^{\ell-1} V_{j}
$$

for $0 \leq j \leq 2^{\ell} \quad\left(\ell \in \mathbb{N}_{0}\right)$ with $U_{j}=Z_{j}(1,1, x)$ and $V_{j}=Z_{j}\left(\frac{1}{x}, \frac{1}{x}, 1\right)$, and in the case $x=1$

$$
w_{2 \ell-j}(1)=\left(\ell^{2}-1\right) Y_{j}-\ell w_{j}
$$

with $Y_{j}=Z_{j}(1,1,1)$. Moreover, a simple consequence of (2.10) with $p=q=1$ as well as $\ell-1$ instead of $\ell$ and (3.3) with $p=r=1$ is

$$
Y_{2^{\iota}-j}=Y_{2^{\ell-1}+j}
$$

for $0 \leq j \leq 2^{\ell-1}$ with $Y_{n}=Z_{n}(1,1,1)$. This equation shows a local symmetry of $Y_{n}$ with respect to the points $n=3 \cdot 2^{\ell-2}(\ell \geq 2)$ (cf. the later Table 3 ).

\section{Further relations and inequalities}

In the following we also admit vanishing coefficients in system (1.1). In order to establish new relations between different solutions $Z_{n}$ we need the definition of a $k$-sequence.

Definition 4.1. Let $k \in \mathbb{N}$ and $\ell \in \mathbb{N}_{0}$.

(i) A finite sequence $\mu_{1}, \mu_{2}, \ldots, \mu_{k}$ is called a $k$-sequence if $\mu_{1} \in\{1,3\}, \mu_{j} \in\{8 \ell+$ $1,8 \ell+3\}$ for $\mu_{j-1}=4 \ell+3$ and $\mu_{j} \in\{8 \ell+5,8 \ell+7\}$ for $\mu_{j-1}=4 \ell+1 \quad(2 \leq j \leq k)$.

(ii) A finite sequence $\mu_{1}, \mu_{2}, \ldots, \mu_{k}, \mu_{k}^{*}$ is called an extended $k$-sequence if $\mu_{1}, \ldots, \mu_{k}$ is a $k$-sequence, $\mu_{k}^{*}=4 \ell+3$ for $\mu_{k}=4 \ell+1$ and $\mu_{k}^{*}=4 \ell+1$ for $\mu_{k}=4 \ell+3$.

The foregoing definitions can be visualized by means of a so-called Collatz graph (cf. [8]). We begin with the directed Collatz graph in Figure 1 for the function $g$ defined by

$$
g(4 \ell+1)=g(4 \ell+3)=2 \ell+1 \quad\left(\ell \in \mathbb{N}_{0}\right)
$$

Inverting the directions and interchanging the neighbouring numbers $4 \ell+1$ and $4 \ell+3$ for all $\ell \in \mathbb{N}_{0}$, we obtain the inversely directed Collat $z$ graph in Figure 2 for the function $f$ defined by

$$
\left.\begin{array}{l}
f(8 \ell+1)=f(8 \ell+3)=4 \ell+3 \\
f(8 \ell+5)=f(8 \ell+7)=4 \ell+1
\end{array}\right\} \quad\left(\ell \in \mathbb{N}_{0}\right)
$$

After these preparations, the numbers of $k$ consecutive vertices in a' directed path of Figure 2 beginning with 1 or 3 , where in the last case the loop at the vertex 3 can be passed several times, yield always terms of a $k$-sequence. The term $\mu_{k}^{*}$ of the.corresponding extended $k$-sequence is fixed by the demand that $\mu_{k}^{*} \neq \mu_{k}$ and that an interchange of 
$\mu_{k}$ and $\mu_{k}^{*}$ again yields an extended $k$-sequence. Note that for all $j$ we have $\mu_{j}<2^{j+1}$.

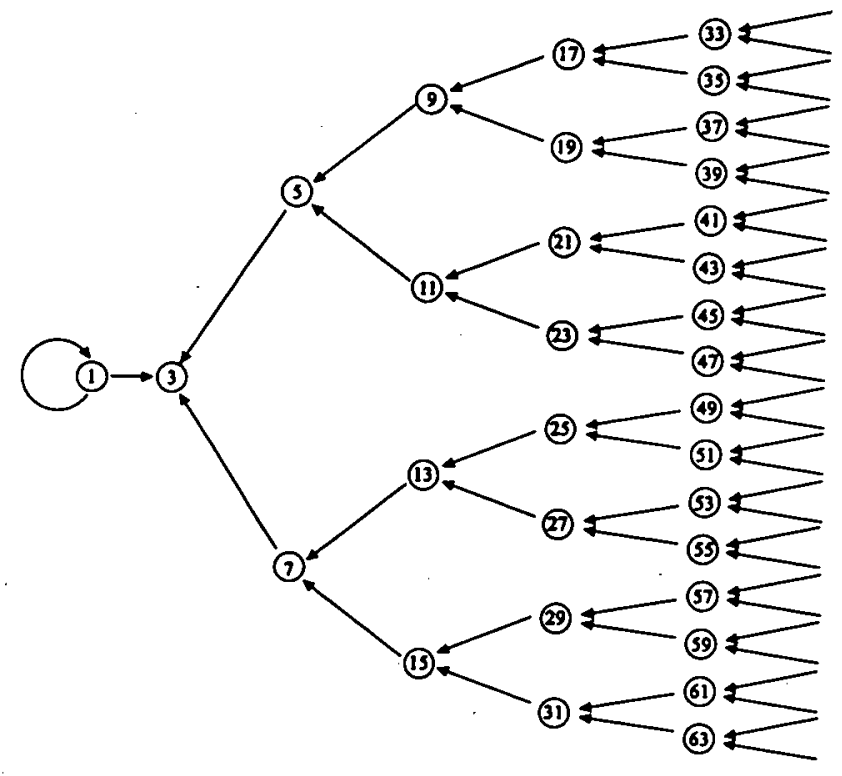

Figure 1: The directed Collatz graph of the function $g$

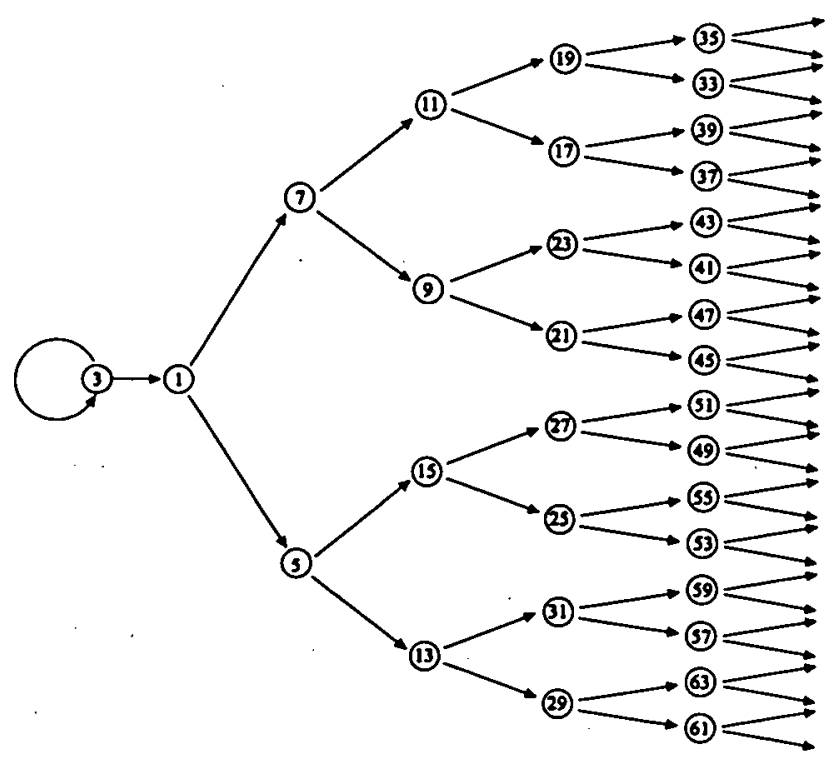

Figure 2: The inversely directed Collatz graph of the function $f$ 
Proposition 4.2. For every extended $k$-sequence the polynomials $Z_{n}$ satisfy the relations

$$
\begin{aligned}
\lambda^{k} Z_{2 n+1}= & \lambda^{k-1} Z_{4 n+\mu_{1}}+\lambda^{k-2} Z_{8 n+\mu_{2}}+\ldots \\
& +\lambda Z_{2^{k} n+\mu_{k-1}}+Z_{2^{k+1} n+\mu_{k}}+Z_{2^{k+1} n+\mu_{k}^{*}}
\end{aligned}
$$

for arbitrary $k \in \mathbb{N}, n \in \mathbb{N}_{0}$ and $\lambda=p+q+r$.

Proof. From system (1.1) we easily derive

$$
\left.\begin{array}{l}
Z_{4 k+1}=p q Z_{k}+r Z_{2 k+1} \\
Z_{4 k+3}=q Z_{2 k+1}+p r Z_{k+1}
\end{array}\right\}
$$

By addition we obtain

$$
(p+q+r) Z_{2 n+1}=Z_{4 n+1}+Z_{4 n+3}
$$

and therefore (4.1) for $k=1$ : If (4.1) is satisfied for a fixed $k$-sequence, we multiply this equation by $\lambda$ and regard that

$$
\begin{aligned}
& \lambda Z_{2^{k+1} n+4 \ell+1}=Z_{2^{k+2} n+8 \ell+1}+Z_{2^{k+2} n+8 \ell+3} \\
& \lambda Z_{2^{k+1} n+4 \ell+3}=Z_{2^{k+2} n+8 \ell+5}+Z_{2^{k+2} n+8 \ell+7} .
\end{aligned}
$$

in view of (4.3). Hence we obtain (4.1) with $k+1$ instead of $k$ and two extended $(k+1)$ sequences, one with the old $\mu_{j}$ for $j \leq k$ and one with the old $\mu_{j}$ for $j \leq k-1$ and $\mu_{k}^{*}$ instead of $\mu_{k}$, and both with suitable $\mu_{k+1}, \mu_{k+1}^{*}$

\section{Remark 4.3.}

1. Further special cases of relations (4.1) besides of (4.3) are

$$
\begin{aligned}
& \lambda^{2} Z_{2 n+1}=\lambda Z_{4 n+1}+Z_{8 n+5}+Z_{8 n+7} \\
& \lambda^{2} Z_{2 n+1}=\lambda Z_{4 n+3}+Z_{8 n+1}+Z_{8 n+3} .
\end{aligned}
$$

2. Dividing (4.1) by $\lambda^{k}$ and considering the case $k \rightarrow \infty$ we obtain the expansion

$$
Z_{2 n+1}=\sum_{\ell=1}^{\infty} \frac{1}{\lambda^{\ell}} Z_{2^{\ell+1} n+\mu_{\ell}}
$$

so long as the series is converging. This is always the case for positive $p, q, r$ but also for some complex coefficients:

Proposition 4.4. The series (4.4) converges for complex $p, q, r$ provided that

$$
C=\max \{|p|,|q|+|r|, 1\}<|\lambda|
$$

where $\lambda=p+q+r$.

Proof. 1. In order to show the convergence of series (4.4) first we shall prove that

$$
\left|Z_{k}\right| \leq C^{\ell}
$$


for $1 \leq k \leq 2^{\ell}\left(\ell \in \mathbb{N}_{0}\right)$. For this reason we shall show by induction that

$$
\left|Z_{2^{\ell}+j}\right| \leq C^{\ell+1}
$$

for $0 \leq j \leq 2^{\ell}$. This inequality is true for $\ell=0, j \in\{0,1\}$ according to $Z_{1}=1 \leq C$ and $\left|Z_{2}\right|=|p| \leq C$. Assume that (4.7) is valid for a fixed $\ell$. Then we have

$$
\begin{gathered}
\left|Z_{2^{\ell+1}+2 j}\right|=|p|\left|Z_{2^{\ell}+j}\right| \leq|p| C^{\ell+1} \leq C^{\ell+2} \\
\left|Z_{2^{\ell+1}+2 j+1}\right| \leq|q|\left|Z_{2^{\ell}+j}\right|+|r|\left|Z_{2^{\ell}+j+1}\right| \leq(|q|+|r|) C^{\ell+1} \leq C^{\ell+2}
\end{gathered}
$$

for $j \leq 2^{\ell}$ and $j<2^{\ell}$, respectively, i.e. (4.7) with $\ell+1$ instcad of $\ell$ so that (4.7) is proved. This implies inequality (4.6) in view of $C \geq 1$.

2. Now, from (4.6) and $\mu_{\ell}<2^{\ell+1}$ we obtain $\left|Z_{2^{\ell+1} n+\mu_{\ell}}\right| \leq C^{\ell+m+1}$ for $n+1 \leq 2^{m}$ in view of $2^{\ell+1} n+\mu_{\ell}<2^{\ell+1}(n+1) \leq 2^{\ell+m+1}$. This yiclds $\left|\frac{1}{\lambda^{\ell}} Z_{2^{\ell+1} n+\mu_{\ell}}\right| \leq C^{m+1}\left(\frac{C}{|\lambda|}\right)^{\ell}$ so that according to (4.5) the series in (4.4) converges

For $k=2^{\ell}+j$ we immediately obtain from (4.7) and $2^{\ell} \leq k \leq 2^{\ell+1}$ :

Corollary 4.5. The polynomials $Z_{k}(k \in \mathbb{N})$ can be estimated by

$$
\left|Z_{k}\right| \leq C k^{c}
$$

with $c=\frac{\ln C}{\ln 2}$.

In the case $p=q=r=1$ we can state the following curious connection between the numbers $Y_{n}=Z_{n}(1,1,1)$ and the Fibonacci numbers $F_{k} \quad\left(k \in \mathbb{N}_{0}\right)$ :

Proposition 4.6. With the notation $m_{k}=\frac{1}{3}\left(2^{k+1}+(-1)^{k}\right)\left(k \in \mathbb{N}_{0}\right)$ the numbers $Y_{m_{k}}=Z_{m_{k}}(1,1,1)$ are the Fibonacci numbers $F_{k}$. These have the extremal property $Y_{n}<Y_{m_{k}}$ for $n<m_{k}$ and $k \geq 2$.

Proof. In view of $m_{0}=m_{1}=1$ and (1.2) the first assertion is valid for $k=0$ and $k=1$. According to

$$
2^{k+1}+(-1)^{k}=2^{k}+(-1)^{k-1}+2\left(2^{k-1}+(-1)^{k-2}\right)
$$

and (1.1) with $p=q=r=1$ the numbers $Y_{m_{k}}$, satisfy the difference equation

$$
Y_{m_{k}}=Y_{m_{k-1}}+Y_{m_{k-2}}
$$

for $k \geq 2$ which proves the first assertion.

In order to prove the second assertion it suffices to consider odd indices since $Y_{2 n}=$ $Y_{n}$ and to consider (4.2) in the specialization

$$
\left.\begin{array}{l}
Y_{4 n+1}=Y_{n}+Y_{2 n+1} \\
Y_{4 n+3}=Y_{n+1}+Y_{2 n+1}
\end{array}\right\} .
$$


The assertion is valid for $n<m_{2}=3$ where $Y_{3}=2$ (cf. Table 3 ). We assume that it is valid for $n<m_{k-1}$ with $k \geq 3$. In the case that $k$ is even we choose $\ell=\frac{1}{3}\left(2^{k-1}-2\right)$ and have

$$
\begin{aligned}
m_{k-2} & =\ell+1 \\
m_{k-1} & =2 \ell+1 \\
m_{k} & =4 \ell+3 .
\end{aligned}
$$

Hence $4 n+1<4 \ell+3$ implies $n \leq \ell$, i.e. $n<\ell+1$ as well as $2 n+1 \leq 2 \ell+1$, and $4 n+3<4 \ell+3$ implies $n<\ell$, i.e. $n+1<\ell+1$ as well as $2 n+1<2 \ell+1$. In the case that $k$ is odd we choose $\ell=\frac{1}{3}\left(2^{k-1}-1\right)$ and obtain

$$
\begin{aligned}
m_{k-2} & =\ell \\
m_{k-1} & =2 \ell+1 \\
m_{k} & =4 \ell+1 .
\end{aligned}
$$

Hence $4 n+1<4 \ell+1$ implies $n<\ell$ as well as $2 n+1<2 \ell+1$, and $4 n+3<4 \ell+1$ implies $n+1 \leq \ell$ as well as $2 n+1<2 \ell+1$. In both cases equations (4.9) and (4.10) show that the second assertion is also valid for $n<m_{k}$ so that it is proved by induction

It can be shown analogously by induction that $Y_{n}<Y_{m_{k}}$ for $m_{k}<n \leq 3 \cdot 2^{k-2}$ and $k \geq 3$, but we can extend this inequality a second time by means of (3.6). Introducing numbers $\bar{m}_{k}(k \in \mathbb{N})$ by $2^{k}-\bar{m}_{k}=2^{k-1}+m_{k}$, i.e. by

$$
\bar{m}_{k}=\frac{1}{3}\left(5 \cdot 2^{k-1}-(-1)^{k}\right)
$$

we have $Y_{m_{k}}=Y_{\bar{m}_{k}}$ according to (3.6). Obviously, $2^{k-1} \leq m_{k} \leq 3 \cdot 2^{k-2} \leq \bar{m}_{k} \leq 2^{k}$ and $m_{k}=\bar{m}_{k}$ if and only if $k=2$, Now, the foregoing remarks and equation (3.6) imply:

Corollary 4.7 For a fixed $k \in \mathbb{N}$ the Fibonacci number $F_{k}$ is equal to the maximum of $Y_{n}$ for $1 \leq n \leq 2^{k}$ which is attained in this interval exactly for both $n=m_{k}$ and $n=\bar{m}_{k}$.

The extremal properties in Proposition 4.6 and in Corollary 4.7 can be checked for the first indices by means of Table 3 where the Fibonacci numbers $Y_{m_{k}}$ are underlined and the Fibonacci numbers $Y_{\bar{m}}$ are labelled by an overhead bar.

\begin{tabular}{r|rrrrrrrrrrrrrrrrrrr|}
$n$ & 1 & 2 & $\mid$ & 3 & 4 & 5 & 6 & 7 & 8 & 1 & 9 & 10 & 11 & 12 & 13 & 14 & 15 & 16 \\
\hline$Y_{n}$ & $\underline{1}$ & $\overline{1}$ & $\mid$ & $\underline{2}$ & 1 & 1 & $\underline{3}$ & 2 & $\overline{3}$ & 1 & 1 & 4 & 3 & $\underline{5}$ & 2 & $\overline{5}$ & 3 & 4 & 1 \\
\hline \hline$n$ & 17 & 18 & 19 & 20 & 21 & 22 & 23 & 24 & 25 & 26 & 27 & 28 & 29 & 30 & 31 & 32 \\
\hline$Y_{n}$ & 5 & 4 & 7 & 3 & $\underline{8}$ & 5 & 7 & 2 & 7 & 5 & $\overline{8}$ & 3 & 7 & 4 & 5 & 1 \\
\hline \hline$n$ & 33 & 35 & 37 & 39 & 41 & 43 & 45 & 47 & 49 & 51 & 53 & 55 & 57 & 59 & 61 & 63 \\
\hline$Y_{n}$ & 6 & 9 & 11 & 10 & 11 & $\underline{13}$ & 12 & 9 & 9 & 12 & $\overline{13}$ & 11 & 10 & 11 & 9 & 6 \\
\hline \hline$n$ & 65 & 67 & 69 & 71 & 73 & 75 & 77 & 79 & 81 & 83 & 85 & 87 & 89 & 91 & 93 & 95 \\
\hline$Y_{n}$ & 7 & 11 & 14 & 13 & 15 & 18 & 17 & 13 & 14 & 19 & 21 & 18 & 17 & 19 & 16 & 11
\end{tabular}

Table 3: The first numbers $Y_{n}=Z_{n}(1,1 ; 1)$ 


\section{Generating functions}

It is useful to construct the generating function

$$
G(t)=\sum_{n=1}^{\infty} Z_{n} t^{n-1}
$$

of the sequence $Z_{n}$. In view of (4.8) the series converges for $|t|<1$. The recursions (1.1) casily imply the functional equation

$$
G(t)=1-r+\left(r+p t+q t^{2}\right) G\left(t^{2}\right)
$$

and therefore by iteration for arbitrary $n \in \mathbb{N}_{0}$

$$
G(t)=(1-r) \sum_{k=0}^{n-1} \prod_{j=0}^{k-1}\left(r+p t^{2^{j}}+q t^{2^{j+1}}\right)+G\left(t^{2^{n}}\right) \prod_{j=0}^{n-1}\left(r+p t^{2^{j}}+q t^{2^{j+1}}\right) .
$$

As usual the products are defined by 1 in the cases $k=0$ and $n=0$. For $|t|<1$ we have $G\left(t^{2^{n}}\right) \rightarrow G(0)=1$ as $n \rightarrow \infty$. Hence for $|t|<1$ we get in the case $|r|<1$

$$
G(t)=(1-r) \sum_{k=0}^{\infty} \prod_{j=0}^{k-1}\left(\dot{r}+p t^{2^{j}}+q t^{2^{j+1}}\right)
$$

and in the case $r=1$

$$
G(t)=\prod_{j=0}^{\infty}\left(1+p t^{2^{j}}+q t^{2^{j+1}}\right) .
$$

However, if we write $(5.2)$ in the form

$$
G(t)-1=p t+q t^{2}+\left(r+p t+q t^{2}\right)\left(G\left(t^{2}\right)-1\right)
$$

we get

$$
G(t)=1+\sum_{k=0}^{\infty}\left(p t^{2^{k}}+q t^{2^{k+1}}\right) \prod_{j=0}^{k-1}\left(r+p t^{2^{j}}+q t^{2^{j+1}}\right)
$$

for arbitrary $r$ and again for $|t|<1$. Summarizing these results we have proved:

Proposition 5.1. For $|t|<1$ the generating function (5.1) has the representation (5.5). In the case $|r|<1$ it can also be represented by (5.3) and in the case $r=1$ by (5.4).

Concerning the different representations (5.4) and (5.5) in the case $r=1 \mathrm{cf}$. [6: p. 233].

Moreover, we consider the generating functions

$$
F(t)=\sum_{n=1}^{\infty} Y_{n} t^{n-1} \quad \text { and } \quad H(t)=\sum_{n=1}^{\infty} w_{n} t^{n-1} .
$$

where $Y_{n}=Z_{n}(1, q, 1)$ and $w_{n}=w_{n}(q)$ so that $F(t)=G(t)$ from (5.1) with $p=r=1$ and (5.2) specializes to

$$
F(t)=\left(1+t+g t^{2}\right) F\left(t^{2}\right)
$$

According to (2.18) and (4.8) the series for $H(t)$ converges for $|t|<1$, too. 
Proposition 5.2. The generating function $H$ from (5.6) satisfies the equation

$$
H(t)=F(t)-1+\left(1+t+q t^{2}\right) H\left(t^{2}\right)
$$

and it can be represented by the series

$$
H(t)=F(t) \sum_{k=0}^{\infty}\left(1-\frac{1}{F\left(t^{2^{k}}\right)}\right)
$$

which converges for $|t|<1$.

Proof. Equation (5.8) follows from (2.16) and (5.6) by straitforward calculations, and (5.9) follows from (5.7) and (5.8) in view of $H(0)=0$ and $F(0)=1$

Let us mention that scries (5.9) can be written in the form

$$
H(t)=\sum_{k=0}^{\infty}\left(F(t)-\frac{F(t)}{F\left(t^{2^{k}}\right)}\right)
$$

where the quotients

$$
\frac{F(t)}{F\left(t^{2^{k}}\right)}=\prod_{j=0}^{k-1}\left(1+t^{2^{j}}+q t^{2^{j+1}}\right)
$$

are polynomials. It is also possible to eliminate $F(t)$ out of (5.7) and (5.8), but then $H\left(t^{4}\right)$ appears in the equation.

\section{Explicit representations}

We begin with very special representations. In our representations we need the dyadic sum-of-digits function $\nu(j)$ and its complement $\mu(k)=\ell-\nu(k)$ for $2^{\ell-1} \leq k<2^{\ell}$ with $j \in \mathbb{N}_{0}$ and $\ell, k \in \mathbb{N}$, i.e. $\nu(j)$ denotes the number of $1 s$ and $\mu(k)$ the number of $0 s$ in the dyadic representation of $j$ resp. $k$. Obviously, we have the initial values $\nu(0)=\mu(1)=0$ and the recursions

$$
\left.\left.\begin{array}{rl}
\nu(2 j) & =\nu(j) \\
\nu(2 j+1) & =\nu(j)+1
\end{array}\right\} \quad \text { and } \quad \begin{array}{rl}
\mu(2 k) & =\mu(k)+1 \\
\mu(2 k+1) & =\mu(k)
\end{array}\right\}
$$

Moreover, we put $\mu(0)=0$ which is compatible with the last equation of (6.1). Since $Z_{2^{\ell k}}=p^{\ell} Z_{k}$ it suffices to consider odd $k$ only.

Proposition 6.1. For $k \in \mathbb{N}$ the polynomials $Z_{2 k+1}$ have the representations

$$
Z_{2 k+1}= \begin{cases}q^{\nu(k)} r^{\mu(k)} & \text { for } p=0 \\ p^{\nu(k)} r^{\mu(k)+1} & \text { for } q=0 \\ p^{\mu(k)} q^{\nu(k)} & \text { for } r=0\end{cases}
$$

Proof. From (1.1) and (4.2) we immediately obtain

$$
Z_{4 k+1}=\left\{\begin{array}{ll}
r Z_{2 k+1} \\
r Z_{2 k+1} \\
p Z_{2 k+1}
\end{array} \quad \text { and } \quad Z_{4 k+3}= \begin{cases}q Z_{2 k+1} & \text { for } p=0 \\
p Z_{2 k+1} & \text { for } q=0 \\
q Z_{2 k+1} & \text { for } r=0 .\end{cases}\right.
$$

In view of $Z_{3}=p r+q$ equations (6.2) are valid for $k=1$. If they are valid for a fixed $k \in \mathbb{N}$, then also for $2 k$ resp. $2 k+1$ instead of $k$ in view of (6.1) and (6.3). Hence the proposition is proved by induction 
Remark 6.2. In view of (1.2) the first and last equations of (6.2) remain valid for $k=0$. In the case $r=0$ we have $Z_{n}=p^{\mu(n)} q^{\nu(n)-1}$ for all $n \in \mathbb{N}$. We even can use Proposition 2.1 for $q=0$ and Proposition 3.1 for $r$. $=0$ if for $j=0$ we interpret $q X_{0}=p-r$ resp. $r U_{0}=p-q$.

In order to deal with the general case we need some preparations. It can easily be seen that

$$
\prod_{j=0}^{\ell-1}\left(1+p t^{2^{j}}\right)=\sum_{k=0}^{2^{\ell}-1} p^{\nu(k)} t^{k}
$$

and, more generally,

$$
\prod_{j=0}^{\ell-1}\left(r+p_{j} t^{2^{j}}\right)=\sum_{k=0}^{2^{\ell-1}} r^{\ell-\nu(k)}\left(\prod_{m=0}^{\nu(k)-1} p_{\gamma_{k m}}\right) t^{k}
$$

where the indices $\gamma_{k m} \in \mathbb{N}_{0}$ are defined by

$$
k=2^{\gamma_{k, \nu(k)-1}}+\ldots+2^{\gamma_{k 1}}+2^{\gamma_{k 0}}
$$

with $\gamma_{k 0}<\gamma_{k 1}<\gamma_{k 2}<\ldots$. For another generalization we need

Definition 6.3. We say that the ordered pair $(i, k) \in \mathbb{N}_{0} \times \mathbb{N}_{0}$ belongs to the relation $\omega(i, k)$, if $i=0$ or if $\left\{\gamma_{i 0}, \gamma_{i 1}, \ldots, \gamma_{i, \nu(i)-1}\right\} \subset\left\{\gamma_{k 0}, \gamma_{k 1}, \ldots, \gamma_{k, \nu(k)-1}\right\}$.

By means of this definition we find that

$$
\prod_{m=0}^{\nu(k)-1}\left(p+q t^{2^{\gamma_{k m}}}\right)=\sum_{\omega(i, k)} p^{\nu(k)-\nu(i)} q^{\nu(i)} t^{i}
$$

Choosing $p_{j}=p+q t^{2^{j}}$ we obtain

$$
\prod_{j=0}^{\ell-1}\left(r+p t^{2^{j}}+q t^{2^{j+1}}\right)=\prod_{j=0}^{\ell-1}\left(r+p_{j} t^{2^{j}}\right)=\sum_{k=0}^{2^{\ell}-1} \sum_{\omega(i, k)} s_{i k \ell} t^{i+k}
$$

according to (6.4) and (6.5) where we have used the abbreviation

$$
s_{i k \ell}=r^{\ell-\nu(k)} p^{\nu(k)-\nu(i)} q^{\nu(i)} .
$$

Proposition 6.4. For $n \in \mathbb{N}$ the solution of problem (1.1) - (1.2) has the representation

$$
Z_{n+1}=\sum_{i+k=n}^{\prime} s_{i k \ell}+\sum_{i+k=n-2^{\ell}}{ }^{\prime} p s_{i k \ell}
$$

where $2^{\ell} \leq n<2^{\ell+1}\left(i, k \in \mathbb{N}_{0}\right)$ and a prime at sums shall mean that $(i, k)$ must belong to $\omega(i, k)$ and that $k \leq 2^{\ell}-1$. 
Proof. Comparing (5.1) with (5.5) we see that $Z_{n+1}$ is the coefficient of $t^{n}$ in the polynomial

$$
\left(p t^{2^{\ell-1}}+q t^{2^{\ell}}\right) \prod_{j=0}^{\ell-2}\left(r+p t^{2^{j}}+q t^{2^{j+1}}\right)+t^{2^{\ell}} \prod_{j=0}^{\ell-1}\left(r+p t^{2^{j}}+q t^{2^{j+1}}\right)
$$

since the product in (5.5) is a polynomial in $t$ of degree $\sum_{j=1}^{k} 2^{j}=2^{k+1}-2$. It is possible to replace $(6.10)$ by

$$
\left(1+p t^{2^{\ell}}\right) \prod_{j=0}^{\ell-1}\left(r+p t^{2^{j}}+q t^{2^{j+1}}\right)
$$

because the difference is a polynomial in $t$ of degree $2^{\ell}-2$ which gives no contribution to the coefficient in question. Now, (6.7) immediately implies (6.9)

\section{Remark 6.5.}

1. In accordance with Corollary 3.2 the first sum of (6.9) is a homogeneous polynomial of degree $\ell$ and the last sum is such a polynomial of degrec $\ell+1$.

2. In view of $i+k=n-2^{\ell}$ and $n<2^{\ell+1}$, the restriction $k \leq 2^{\ell}-1$ is automatically satisfied in the second sum of (6.9).

By means of (2.11), it follows from (6.9) with $q=p x$ :

Corollary 6.6. For $n \in \mathbb{N}$ the polynomial $w_{n+1}$ has the representation

$$
w_{n+1}(x)=\ell \sum_{i+k=n} x^{\nu(i)}+(\ell+1) \sum_{i+k=n-2^{\ell}}{ }^{\prime \nu(i)}
$$

with the same restrictions as in Proposition 6.4 .

Comparing (6.9) and (6.11) with (2.18) in the case $p=r=1, x=q$ and $n=2^{\ell}+j-1$ we obtain the simplification

$$
Y_{j}=\sum_{i+k=j-1}{ }^{\nu(i)}
$$

where $j \in \mathbb{N},(i, k) \in \omega(i, k)$ and $Y_{j}=Z_{j}(1, q, 1)$, but a further restriction with respect to $k$ is not required.

In the special case $r=1$ we can derive another type of representations. For convenience we use the notation $z_{n}=z_{n}(p, q)=Z_{n}(p, q, 1)$ for $n \in \mathbb{N}_{0}$. If we introduce new parameters $\alpha$ and $\beta$ as solutions of $\xi^{2}-p \xi+q=0$ so that

$$
\left.\begin{array}{l}
p=\alpha+\beta \\
q=\alpha \beta
\end{array}\right\}
$$

we can write system (1.1) with $r=1$ in the form

$$
\begin{aligned}
z_{2 k} & =(\alpha+\beta) z_{k} \\
z_{2 k+1} & =\alpha \beta z_{k}+z_{k+1}
\end{aligned}
$$

and every $z_{n}$ is a symmetric polynomial with respect to $\alpha$ and $\beta$. The generating function (5.4) supplies a representation for $z_{n}$ : 
Proposition 6.7. The polynomial $z_{n}$ has the representation

$$
z_{n}=\sum_{j=0}^{n-1} \alpha^{\nu(j)} \beta^{\nu(n-1-j)}
$$

where $\alpha$ and $\beta$ are determined by (6.13) and $\nu(j)$ by (6.1).

Proof. In view of (6.13) we have $1+p t+q t^{2}=(1+\alpha t)(1+\beta t)$ so that the generating function (5.4) has the form

$$
G(t)=\prod_{j=0}^{\infty}\left(1+\alpha t^{2^{j}}\right) \prod_{j=0}^{\infty}\left(1+\beta t^{2^{j}}\right)
$$

for $|t|^{\circ}<1$. Owing to

$$
\prod_{j=0}^{\infty}\left(1+\xi t^{2^{j}}\right)=\sum_{k=0}^{\infty} \xi^{\nu(k)} t^{k}
$$

we obtain

$$
G(t)=\sum_{j=0}^{\infty} \alpha^{\nu(j)} t^{j} \sum_{k=0}^{\infty} \beta^{\nu(k)} t^{k}
$$

and hence, by means of the Cauchy product and (5.1), representation (6.14)

Solving (6.13) with respect to $p$ and $q$ it is possible in (6.14) to express $z_{n}$ explicitly by means of the parameters $p$ and $q$.

\section{Examples 6.8.}

1. In the special case $\beta=1$ and thercforc $\alpha=q, p=q+1$ formula (6.14) reduces to a representation of $S_{n}=z_{n}(q+1, q)$ in [2].

2. In the special case $q=1$, i.c. $\beta=\frac{1}{\alpha}$, formula (6.14) simplifies to

$$
z_{n}(p, 1)=\sum_{j=0}^{n-1} \alpha^{\nu(j)-\nu(n-1-j)}
$$

where

$$
\alpha=\frac{p}{2} \pm \sqrt{\frac{p^{2}}{4}-1}
$$

and, in particular for $p=2$, i.e. $\alpha=1,(6.16)$ implies $z_{n}(2,1)=n$ which also follows immediately from (1.1) with $p=2, q=r=1$ and (1.2). For $p \geq 2$ we can put $p=2 \cosh r$ with real $r$ so that $\alpha=e^{ \pm r}$ and

$$
z_{n}(2 \cosh r, 1)=\sum_{j=0}^{n-1} \cosh [r(\nu(j)-\nu(n-1-j))] .
$$

For $-2 \leq p \leq 2$ we can put $p=2 \cos \varrho$ with real $\varrho$ so that $\alpha=e^{ \pm i \varrho}$ and

$$
z_{n}(2 \cos \rho, 1)=\sum_{j=0}^{n-1} \cos [\varrho(\nu(j)-\nu(n-1-j))] .
$$


Of course, representations (6.18) and (6.19) are also valid for complex $r$ resp. $\varrho$.

3. A last special case is $p=1$ which concerns the polynomials $Y_{n}=Z_{n}(1, q, 1)=$ $z_{n}(1, q)$. Formula $(6.14)$ yields the representation

$$
Y_{n}=\sum_{j=0}^{n-1} \alpha^{\nu(j)}(1-\alpha)^{\nu(n-1-j)}
$$

where

$$
\alpha=\frac{1}{2} \pm \sqrt{\frac{1}{4}-q}
$$

From (2.18) and (6.20) also a representation for $w_{n}$ can be obtained, but we do not deal with that case.

Finally, we want to give a third type of representation for $Y_{n}=Z_{n}(1, \dot{q}, 1)=z_{n}(1, q)$ where once more it suffices to consider odd $n$ only. From $(2.10)$ with $p=r=1$ and (2.18) with $x=q$ we obtain

$$
Y_{2^{\ell}+2^{\lambda}+j}=(q(\ell-\lambda)+1) Y_{2^{\lambda}+j}-q Y_{j}
$$

for $0 \leq j \leq 2^{\lambda}<2^{\ell}$. As in (6.5), an arbitrary positive odd integer can be written in the form

$$
n_{k}=2^{\gamma_{k}}+2^{\gamma_{k-1}}+\ldots+2^{\gamma_{1}}+2^{\gamma_{0}}
$$

with $\gamma_{0}=1<\gamma_{1}<\gamma_{2}<\ldots\left(k \in \mathbb{N}_{0}\right)$ and $\gamma_{j} \in \mathbb{N}$. For a fixed sequence $\gamma_{j}$ we introduce the notation

$$
\eta_{j}=q\left(\gamma_{j}-\gamma_{j-1}\right)+1 \quad(j \in \mathbb{N})
$$

Then, with $\ell=\gamma_{k}, \lambda=\gamma_{k-1}$ and $j=n_{k-2},(6.22)$ can be written as

$$
Y_{n_{k}}=\eta_{k} Y_{n_{k-1}}-q Y_{n_{k-2}}
$$

for $k \geq 2$. Since $n_{0}=1$ and $n_{1}=2^{\gamma_{1}}+1$ we have the initial values $Y_{n_{0}}=1$ and $Y_{n_{1}}=q \gamma_{1}+1=\eta_{1}$; cf. (2.10) with $p=j=1$ and $\ell=\gamma_{1}$.

Proposition 6.9. For $k \in \mathbb{N}_{0}$ the polynomial $Y_{n}=Z_{n}(1, q, 1)$ has the representation

$$
Y_{n_{k}}=\sum_{j=0}^{[k / 2]}(-q)^{j} \eta_{i_{1}} \eta_{i_{2}} \cdots \eta_{i_{k-2 j}}
$$

where the sum runs over all indices with $1 \leq i_{1}<i_{2}<\ldots \leq k$ under the condition that $i_{j}$ is odd or even for $j$ odd or even, respectively.

Proof. In view of the initial values, (6.24) is valid for $k=0$ and $k=1$. If $(6.24)$ is valid up to a fixed $k$, then we obtain from (6.23)

$$
Y_{n_{k+1}}=\sum_{j=0}^{[k / 2]}(-q)^{j} \eta_{i_{1}} \eta_{i_{2}} \cdots \eta_{i_{k-2 j}} \eta_{k+1}+\sum_{j=1}^{[(k+1) / 2]}(-q)^{j} \eta_{i_{1}} \eta_{i_{2}} \cdots \eta_{i_{k+1-2 j}}
$$

and these sums can be gathered up as one single sum (6.24) with $k+1$ instead of $k$ since $i_{k+1-2 j} \leq k-1$ in the second sum 
The first sums (6.24) with $k \geq 2 \mathrm{read}$

$$
\begin{aligned}
& Y_{n_{2}}=\eta_{1} \eta_{2}-q \\
& Y_{n_{3}}=\eta_{1} \eta_{2} \eta_{3}-q\left(\eta_{1}+\eta_{3}\right) \\
& Y_{n_{4}}=\eta_{1} \eta_{2} \eta_{3} \eta_{4}-q\left(\eta_{1} \eta_{2}+\eta_{1} \eta_{4}+\eta_{3} \eta_{4}\right)+q^{2}
\end{aligned}
$$

By means of (2.18) and (6.24) it is possible to derive also a representation for $w_{n_{k}}$ but we are not concerned with that.

\section{References}

[1] Berg, L.: Behandlung einer Funktionalgleichung. Wiss. Z. Univ. Rostock 5, Math.-Nat. Reihe 3 (1955/56), 367 - 372.

[2] Berg, L. and M. Krüppel: De Rham's singular function, two-scale difference equations and Appell polynomials. Results in Math. (to appear).

[3] Berg, L. and M. Krüppel: On discrete solutions of two-scale difference equations (to appear).

[4] Daubeschies, I.: Ten Lectures on Wavelets. Philadelphia: SIAM 1992.

[5] Jentsch, W.: Theoretische und numerische Auflösung eines Systems unendlich vieler Gleichungen mit unendlich vielen Unbekannten. Wiss. Z. Hochsch. Elektrotech. Ilmenau 5 (1959)3, $113-119$.

[6] Knopp, K.: Theorie und Anwendung der unendlichen Reihen. Berlin: Springer-Verlag 1964.

[7] Micchelli, C. A.: Mathematical Aspects of Geometric Modeling. Philadelphia: SIAM 1995.

[8] Wirsching, G. J.: The Dynamical System Generated by the $3 n+1$ Function. Lect. Notes Math. 1681 (1998), $1-158$.

Received 18.01.2000; in revised form 17.07.2000 